University of Nebraska - Lincoln

DigitalCommons@University of Nebraska - Lincoln

Timothy J. Gay Publications

Research Papers in Physics and Astronomy

2002

\title{
Progress with Optically Pumped Sources of Polarized Electrons
}

Mark A. Rosenberry

University of Nebraska-Lincoln, mrosenberry@siena.edu

Herman Batelaan

University of Nebraska - Lincoln, hbatelaan@unl.edu

J. P. Reyes

University of Nebraska - Lincoln

Timothy J. Gay

University of Nebraska - Lincoln, tgay1@unl.edu

Follow this and additional works at: https://digitalcommons.unl.edu/physicsgay

Part of the Physics Commons

Rosenberry, Mark A.; Batelaan, Herman; Reyes, J. P.; and Gay, Timothy J., "Progress with Optically Pumped Sources of Polarized Electrons" (2002). Timothy J. Gay Publications. 54.

https://digitalcommons.unl.edu/physicsgay/54

This Article is brought to you for free and open access by the Research Papers in Physics and Astronomy at DigitalCommons@University of Nebraska - Lincoln. It has been accepted for inclusion in Timothy J. Gay Publications by an authorized administrator of DigitalCommons@University of Nebraska - Lincoln. 


\title{
Progress with Optically Pumped Sources of Polarized Electrons
}

\author{
M.A. Rosenberry, H. Batelaan, J.P. Reyes, T.J. Gay \\ Behlen Laboratory of Physics, University of Nebraska, Lincoln, NE 68588-0111
}

\begin{abstract}
We report our work in developing new "turn-key" sources of polarized electrons. These sources operate by extracting the electrons from a discharge and polarizing them through optical pumping. Preliminary work demonstrates that beams of $4 \mu \mathrm{A}$ with greater than $20 \%$ polarization are possible. Such devices could enormously simplify the running of many experiments in the fields of atomic structure, magnetic materials and biophysics.
\end{abstract}

\section{INTRODUCTION}

Polarized electrons are an extremely valuable tool in physics, and are used to study electron-atom scattering (e.g. in plasmas) [1], magnetism at surfaces (e.g. in nanostructures) [2], and the chiral structure of molecules [3]. At present the standard source of polarized electrons is a specially prepared GaAs crystal. A typical GaAs system produces currents of $20 \mu \mathrm{A}$ with $30 \%$ polarization, though special crystals and extensive preparations can increase this to $100 \mu \mathrm{A}$ at $70 \%$ polarization [4]. Such performance can also be obtained with a properly constructed flowing $\mathrm{He}^{*}$ afterglow source [5].

Both of these sources have stringent vacuum requirements, so that maintenance of the system is time-consuming. More importantly, they are difficult to use, so that extensive training of their operating personnel is necessary. We have been developing sources of polarized electrons that operate at pressures of about a torr and require minimal operator sophistication. With the proper equipment, our source would be "turn-key" (i.e. trivial to operate) while providing an equivalent electron beam to present standard sources.

\section{RUBIDIUM SPIN-EXCHANGE DEVICE}

The body of our system is a Conflat ${ }^{\mathrm{TM}} 23 / 4$ " nipple that is 6 " in length. To this we have attached inlets for rubidium, discharge gas, and the $400 \mathrm{~V}$ needed to run a cold cathode discharge. The system is heated to around $80{ }^{\circ} \mathrm{C}$, giving a rubidium density of about $10^{12}$ per cc. The source chamber also contains between 0.4 and 2.0 torr of discharge gas $\left(\mathrm{N}_{2}, \mathrm{He}, \mathrm{Ar}\right.$ or some combination). A weak electric field $(1 \mathrm{~V} / \mathrm{cm})$ and a moderate magnetic field $(400 \mathrm{G})$ act to steer the electrons towards the target region. En route, the electrons pass through the polarized rubidium vapor and undergo spin-

CP604, Correlations, Polarization, and Ionization in Atomic Systems edited by D. H. Madison and M. Schulz

(C) 2002 American Institute of Phvsics 0-7354-0048-2/02/\$19.00 


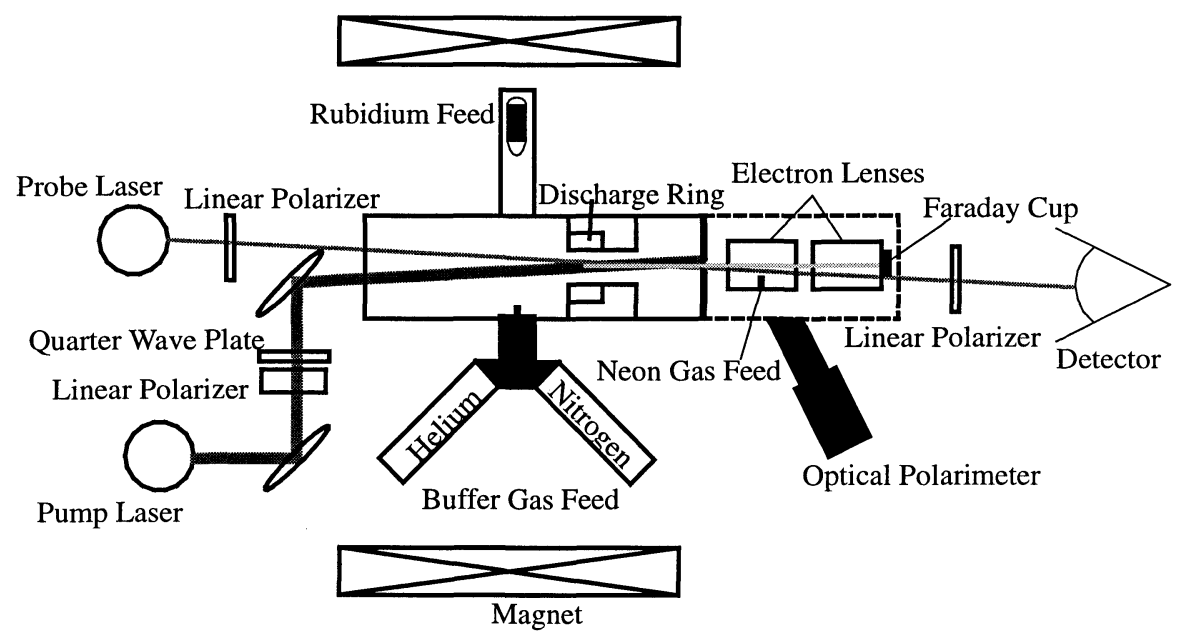

Figure 1: Rubidium Spin Exchange Apparatus

exchange collisions, causing them to acquire some of the rubidium polarization.

The source chamber is separated from the target region by an aperture of $0.9 \mathrm{~mm}$, giving a pressure ratio of about $10^{4}$. The target region also contains $\mathrm{He}$ or $\mathrm{Ne}$ at a pressure of $10^{-4}$ torr, which is excited by the extracted electron beam. The energy width of the electron beam (about $1 \mathrm{eV}$ ) can be estimated from the target gas excitation function. The polarization of the light emitted by the target atoms is also used to determine the electron polarization through known formulae [6]. The electrons then continue along our lens train to a Faraday cup where the current is measured.

This system uses both a pump and a probe laser. The probe beam is at the Rb D2 transition $(780 \mathrm{~nm})$, and is used to determine the rubidium density and polarization through the optical Faraday rotation. At larger values of B $(200 \mathrm{G})$, the rotation is dominated by the diamagnetic effect and determines the density, while at low values $(20 \mathrm{G})$ the rotation is due to the paramagnetic effect and measures the polarization. The equations are given below, with $\delta$ being the detuning from resonance, $\Delta \varphi$ the rotation, $L$ the cell length of $15 \mathrm{~cm}$, and $\Gamma$ is the natural linewidth of $5.4 \mathrm{MHz}$.

$$
\begin{aligned}
\eta_{\mathrm{Rb}} & =\frac{8 \pi \delta^{2}(\Delta \varphi)}{\mathrm{L} \Gamma \lambda^{2} \mu_{\mathrm{B}} \mathrm{B}} ; \\
\mathrm{P}_{\mathrm{Rb}} & =\frac{56 \pi \delta(\Delta \varphi)}{3 \eta_{\mathrm{Rb}} L \Gamma \lambda^{2}}
\end{aligned}
$$

The pump beam (at the D1 transition) optically pumps the rubidium [7]. For preliminary work we employed a $250 \mathrm{~mW}, 40 \mathrm{GHz}$ wide dye laser for the pump beam. This device was highly temperamental, making "turn-key" operation impossible. We have since moved to a $10 \mathrm{~W}$ diode bar, which is temperature controlled with a homebuilt circuit. We have narrowed the frequency profile of this laser (see Figure 2) 


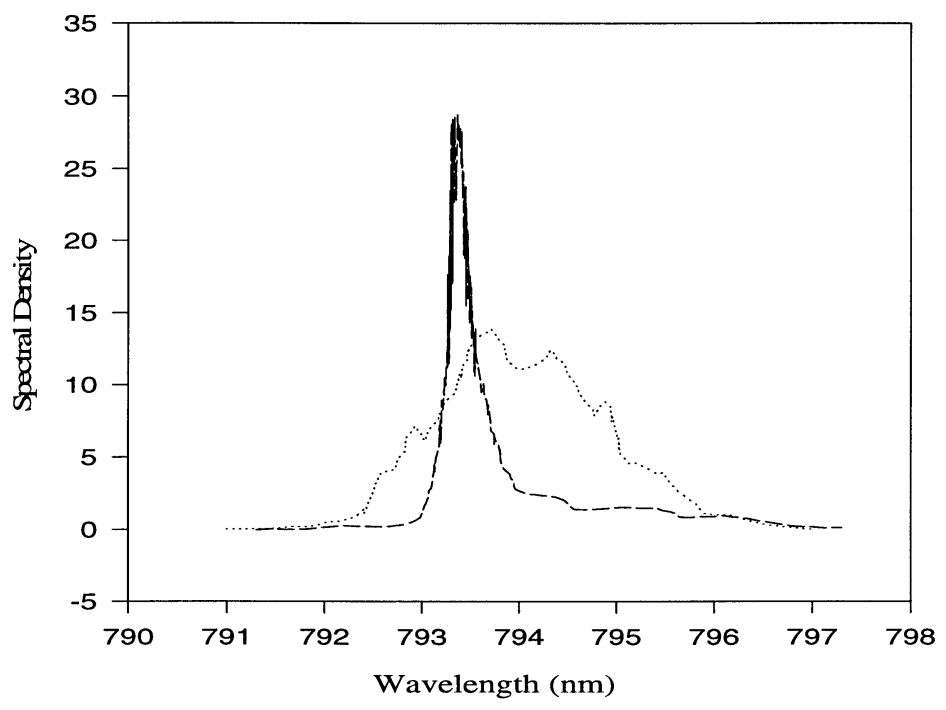

Figure 2: Narrowing with Grating Feed-back

according to the method of Walker et al. [8]. Once set up, this device provides the resonant light needed with only a few seconds of tweaking, as well as generating better rubidium polarizations than the dye laser.

We are also investigating the roles of the buffer gas. To begin with, the buffer gas determines polarization of the electrons. It does so by pressure broadening the rubidium absorption line, reducing the diffusion rates for both $\mathrm{Rb}$ and electrons, and moderating the energy of the electrons (which affects the spin exchange cross-section). The buffer gas also sets the discharge characteristics, which determine the current. Figure 3 shows how these effects compete: the rubidium polarization (which is proportional to the electron polarization) rises steadily with buffer gas pressure, while the current drops precipitously as the density increases.

Different buffer gases may affect the system in different ways. $\mathrm{N}_{2}$ is the standard buffer gas for $\mathrm{Rb}$ optical pumping, since its vibrational levels allow it to effectively quench excited $\mathrm{Rb}$ to prevent radiation trapping [7]. Our buffer gas pressures $(<1$ torr) are sufficiently low that quenching effects are minimal, freeing us to consider other gases. For example, electrons diffuse through helium more readily than nitrogen, so that our currents improve when using a helium buffer gas. The rubidium polarization is somewhat reduced, but the overall figure of merit $\mathrm{P}^{2} \mathrm{I}$ is still improved. Our best results to date (using the dye laser) were obtained in helium: $4 \mathrm{uA}$ with $23 \%$ polarization [7].

Argon, which has a Ramsauer minimum in its cross section, yields even larger currents and should give even better results. Alternately, a mix of gases could be used: nitrogen to moderate the electron energies and a heavy noble gas to improve optical pumping. Unfortunately we have been unable to test these hypotheses due to two 


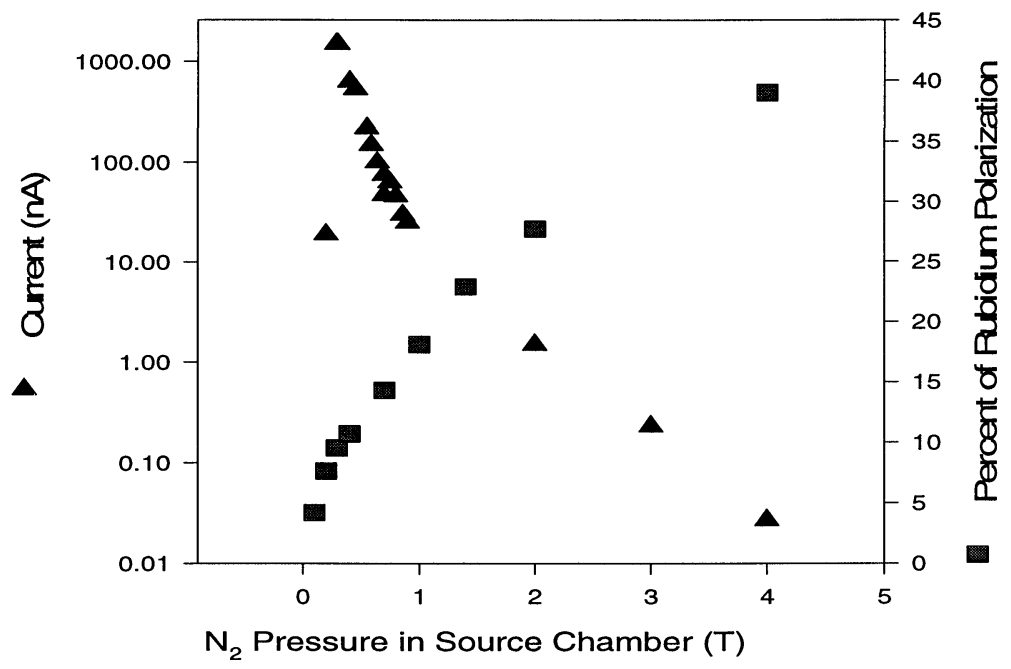

Figure 3: Current and Rubidium Polarization vs Nitrogen Pressure

difficulties. The first problem is the discharge, which can switch between different modes that have different characteristics. This has made systematic studies difficult. To overcome this, we are therefore building a new system that will generate the electrons with a filament, then polarize them through spin exchange. Work on this modification is in progress.

The other obstacle is that for some reason, we have been unable to produce electron polarizations of more than $2 \%$ using the diode laser. This has been very puzzling, but are hopeful that once the problem is resolved, the improved laser power and new gas mix will lead to a substantial improvement in the performance, making it nearly comparable with present standards.

\section{METASTABLE NOBLE GAS SOURCE}

We have also been developing a second possible source of polarized electrons. This system extracts polarized electrons directly from optically-pumped metastable atoms rather than through an intermediate species. It is therefore even easier to use than the one based on spin exchange with rubidium. Studies of this technique have been performed in the past using He, but resulted in only moderate performance; typically currents of tens of $\mu \mathrm{A}$ with less than $10 \%$ polarization [10].

It is not known exactly why such low values are obtained. However, one problem is certainly the difficulty in producing sufficiently large metastable densities while limiting the production of spurious (unpolarized) electrons. Our work leads us to believe that the key parameter is the ratio of metastable density to that of the ground state atoms. This parameter is crucial in setting the percentage of extracted electrons 
that have undergone a polarizing interaction. Therefore heavier noble gases, where these ratios are larger, should generate much better beams that $\mathrm{He}^{*}$.

To test this hypothesis we are developing a system that uses either argon or krypton instead of helium. The apparatus is extremely simple, being a glass tube connected to a small turbo pump via an aperture. Metal bands around the outside the cell create an RF discharge, while pump and probe lasers are used as described above. Typical discharge pressures are $30 \mathrm{mTorr}$ of gas, and it is hoped that cleanliness requirements will not be as stringent as those for helium.

We have confirmed by direct measurement that the ratio (of metastable to ground state atoms) is better in $\mathrm{Ar}$ than that of $\mathrm{He}$ by an order of magnitude (see Figure 4). We are now in the process of determining how these modifications improve the electron polarization.

To conclude, our new source that relies on spin exchange with optically pumped rubidium to polarize our electron beam. This source shows great promise of being far easier to use and maintain than a GaAs crystal, making it highly desirable for many applications.

This work has been supported by NSF grant \#PHY-9704650.

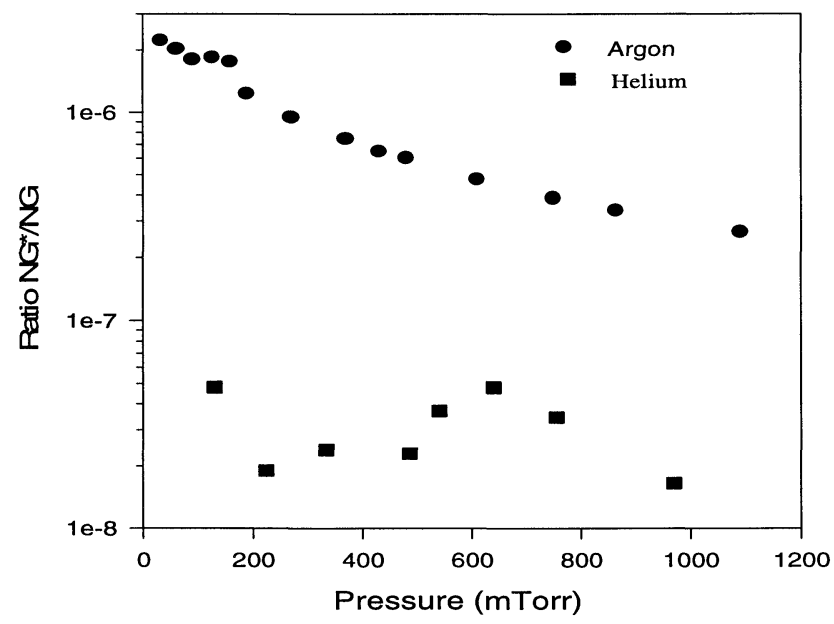

Figure 4: Metastable Fractions 


\section{REFERENCES}

1. H.M. Al-Khateeb, B.G. Birdsey, and T.J. Gay, Phys. Rev. Lett. 85, 4040 (2000)

2. See, e.g. M. Johnson, Science 260, 320 (1993)

3. K.W. Trantham, M.E. Johnston, and T.J. Gay, J. Phys. B. 28, L543 (1995)

4. Polarized Gas Targets and Polarized Beams, edited by R.J. Holt and M.A. Miller, AIP Conf. Proc. No. 421 (AIP, New York, 1998)

5. G.H Rutherford et. al., Rev. Sci Instrum. 61, 1460 (1990)

6. K. Trantham, M.S. thesis at University of Missouri-Rolla, 1993

7. W. Happer, Rev. Mod. Phys. 44169 (1972)

8. B. Chann, I. Nelson, T.G. Walker, "Frequency-Narrowed External Cavity Diode Laser Array Bar", preprint available at www-atoms.physics.wisc.edu

9. H. Batelaan, A.S. Green, B.A. Hitt, and T.J. Gay, Phys. Rev. Lett. 82, 4216 (1999)

10. Ray Vandiver, Ph.D. Thesis, University of Missouri-Rolla, 1993

M.V. McCusker,L.L Hadfield, and G.K. Walters , Phys. Rev. A 5177 (1972) 